\title{
Premature Ovarian Insufficiency: Is it te Same as Menopause?
}

The terms premature ovarian insufficiency (POI) and menopause are often used synonymously, yet they are different and merit management as separate entities. The characteristic feature of POI is amenorrhea in women less than 40 years of age with biochemical evidence of hypoestrogenism and elevated serum gonadotropin levels. It is either a primary ovarian defect with absence of menarche or presents with secondary amenorrhea before the age of 40. Secondary amenorrhea can be due to arrested follicular development or premature depletion of ovarian follicles. Spontaneous resumption of ovarian function is not rare nor it is a brief phenomenon in these women. Majority of women with POI have intermittent ovarian function and unlike menopausal women 3-5\% may become pregnant.

The incidence of POI has been variously reported at $1-4 \%$ with the rising figures with increasing age from one case in 1000 women at 30 years to one per 250 women at 35 to $1 \%$ by the age of 40 years and $10 \%$ before 45 years. Pathophysiologically, POI can be subdivided into four nonpermanent interchangeable clinical states starting with occult POI with unexplained subfertility and failed superovulation despite normal FSH. On progression to biochemical POI a similar presentation with raised FSH is evident. The third phase presents with varied menstrual disturbance like oligo or polymenorrhea or metorrhagia with elevated FSH followed by the final extreme state of primordial follicle depletion with amenorrhea, infertility and elevated menopausal gonadotropins. For most women, it is an unexpected diagnosis with unpleasant symptoms. The most distressing issue is the loss of fertility considered the essence of femininity by many. The ovary has either an absence of follicles or any remaining follicles fail to respond to stimulation.

Although it is said that the age of menopause is not something women can intentionally change, high dietary intake of vitamin D and calcium from dairy foods as well as vegetable protein intake may be associated with a lower risk for early menopause. The etiology of POI is idiopathic in two thirds of women while familial or genetic factors contribute to $20 \%$, autoimmune $10 \%$ and a small proportion due to iatrogenic reasons like chemotherapy, irradiation, smoking, increased use of gonadotropin stimulations and ovarian surgery. The combination of low estradiol and high gonadotropin levels defines POI. Two serum FSH levels in menopausal range at least one month apart are considered diagnostic. The assessment of serum LH can assist in differentiating the probable cause. The LH levels markedly elevated in autoimmune oophritis and FSH is mildly elevated. In contrast with spontaneous idiopathic POI the serum FSH is notably higher than LH levels. Autoimmune screening can start with simple complete blood count, ESR, blood glucose testing and serum electrolytes followed by TSH, ANA, RA factor and ovarian antibody as indicated. In the very young patient, karyotype and test for fragile $\mathrm{X}$ chromosome are considered.

Women with POI are at increased risk of morbidity due to low estrogen status related to cardiovascular, neurological, psychiatric sequelae and osteoporosis. They are also at higher risk of various autoimmune endocrine conditions like diabetes, as well as nonendocrine diseases including pernicious anemia and rheumatoid arthritis. The use of unproven fertility treatments can cause serious harm as does the associated depression. Although there is a paucity of large long-term follow-up studies on POI, there is limited evidence showing increased all cause as well as stroke-related mortality in women with POF before 40 years.

POI over time has cumulative negative effects. These can be mitigated by timely diagnosis and initiating emotional support, healthy lifestyle measures and hormone replacement therapy at least till the physiological age of menopause around 52 years. The use of hormones in this age group is considered safe as the estrogen years are not increased. Many advocate that estrogen be prescribed in higher doses than in postmenopausal women to ensure adequate estrogenisation of vaginal epithelium and maintain age appropriate bone density. An important caveat is that hormone replacement therapy does not prevent ovulation or pregnancy in these women with 5-10\% chance of spontaneous pregnancy. The The North American Menopause Society (NAMS) HRT statement 2017 has changed from 'lowest dose for the shortest period of time' to a more pragmatic concept of 'appropriate dose, duration, regimen, and route of administration' fits well to the POI woman.

To optimize the long-term use of HRT in these women, use of transdermal route of estrogens to minimize hepatic first-pass metabolism can be considered. For endometrial protection safer progetogen options include 
micronized progesterone, Mirena IUS as well as the SERM, bazedoxifene while tibolone by itself is a time-tested agent useful for its mix of estrogenic, progestogenic and androgenic effects in women with POI. The use of DHEA supplements by increasing the expression of a receptor in preovulatory granulosa cells in women with premature ovarian failure may increase the chances of getting pregnant, reduce the risk of miscarriages, and makes IVF more successful. Yet, recent recommendations suggest that it should not be used in patients of POF with normal adrenal function.

Women should avoid unproven treatments to restore fertility as "sometimes the remedy is worse than the disease." ART, oocyte donation, embryo donation, surrogacy, ovarian cryopreservation in iatrogenic POF and adoption are the options in clinical practice. Research on mesenchymal stem cell transplantation for ovary and uterus are in the pipeline and results are awaited.

In conclusion women experiencing premature menopause (before age 40 years) or early menopause (between ages 40 and 45 years) are at a higher risk of overall mortality as well as increased incidence of cardiovascular, neurological, psychiatric diseases and osteoporosis. Most of these adverse outcomes are prevented by estrogen replacement yet, not all long-term consequences are preventable implicating other hormonal mechanisms.

\author{
Syeda Batool Mazhar \\ Professor and Head \\ $\mathrm{MCH}$ Centre \\ Pakistan Institute of Medical Sciences \\ Islamabad, Pakistan \\ Ruchika Garg \\ Associate Professor \\ Department of Obstetrics and Gynecology \\ SN Medical College, Agra \\ Uttar Pradesh, India
}

\title{
REFERENCES
}

1. Shuster LT, Rhodes DJ, Gostout BS, Grossardt BR, Rocca WA. Premature menopause or early menopause: Long term health consequences. Maturitas. 2010;161-166.

2. Bachelot A, Nicolas C, Bidet M, Dulon J, Leban M, Golmard JL, Polak M, Touraine P. Long-term Outcome of Ovarian Function in Women With Intermittent Premature Ovarian Insufficiency. Clin Endocrinol. 2017;86(2):223-228.

3. The 2017 hormone therapy position statement of The North American Menopause Society Menopause. 2017;24(7):728-753. 